\title{
Mobile apps for Mental Health: a content analysis
}

\author{
Md. Aminul Islam ${ }^{1}$, Naziat Choudhury ${ }^{2}$ \\ ${ }^{1}$ Faculty, Department of Media Studies and Journalism, University of Liberal Arts Bangladesh (ULAB). \\ ${ }^{2}$ Associate Professor, Department of Mass Communication and Journalism, University of Rajshahi, \\ Bangladesh. \\ Corresponding author: Md. Aminul Islam. \\ Email - aminul.vu@gmail.com / aminul.islam@ulab.edu.bd
}

\begin{abstract}
Background: The mobile phone is arguably one of the fastest spreading and adopted technologies in the world. Various applications (Apps) and their uses are relatively new in health care, their usages are increasing phenomenally. This paper is aimed at analyzing different features of mental health-related apps. Methodology: This study examines multiple dimensions of mental health-related apps available in Google Play Store for four years between 2016 and 2020. We used a set of keywords such as mental health, mental illness, mental disorders, and the cure of mental disorder and the healing of mental illness to search apps available on the play store. We scrapped the data using various software and programming tools.

Results: Our results show that mental health Apps mainly deal with following symptoms: depression, anxiety, general mental health, stress, post traumatic-stress disorder, bipolar disorder, panic disorder, sleep disorder, schizophrenia, obsessive-compulsive disorder, substance abuse (drug and alcohol), addiction (technology, etc). On the other hand, Apps offer various approach to improve mental health. The approaches include relaxation, stress management, symptom tracking, calming audio, keeping a diary, connect with mental health resources, interpersonal support, meditation, and mood tracking.

Conclusion: These easy to use and interactive mental health apps dealt with specific mental health concerns. Relaxation was the common technique used to deal with these concerns. It was difficult to predict the trustworthiness these apps by looking at their rating and the number of people who rated.
\end{abstract}

Keywords: Mental Health, Apps, Smart-Phone, Depression, common mental disorders, approaches to mental wellbeing.

(Paper received $-5^{\text {th }}$ July 2020, Peer review completed $-5^{\text {th }}$ August 2020, Accepted $-6^{\text {th }}$ August 2020)

\section{INTRODUCTION}

The prevalence of common mental disorders, a range of anxiety and depressive disorders which include depression, generalised anxiety disorder (GAD), panic disorder, phobias, social anxiety disorder, obsessivecompulsive disorder (OCD) and post-traumatic stress disorder (PTSD), is on the rise across the world [1], becoming a global public health concern [2]. People from different regions and ages [3] are living with the burden of some form of the disorders which include mood disorders (such as depression or bipolar disorder), anxiety disorders, personality disorders, psychotic disorders (such as schizophrenia), eating disorders, trauma-related disorders (such as post-traumatic stress disorder), substance abuse disorders and self-harm [4-5]. Various social stigmas are among the key factors that prevent people to seek mental health care services [6] at hospitals and private consultation chambers. To avoid being stigmatized, many people suffer silently without availing treatment and rely on self-care methods. Various studies indicate that Smartphone Apps are becoming popular as a tool to facilitate self-care [7]. As researches suggest that there is a strong association between mental health and social taboos and stigmas, people are finding mental health apps as an alternative tool to seek help [8-9]. Meanwhile, another study found that Smartphone Apps are effective tools for improving mental health and wellbeing [10-11]. This paper attempts to provide a summary of the existing mental health apps to understand the common areas of mental disorder that are focused by app 
developers. The apps are being highly rated are explored here as rating options are used to provide a critical review of the quality of apps.

Mobile phone applications (app) are a specialized feature of the mobile phone. The focus of these apps covers a vast area of interest. One of the tools that have been used to provide some support along with face-to-face interventions is these apps. In creating awareness and in building support for mental health issues, apps have been known to play a significant role [12]. The ease of use, accessibility, and convenience have contributed to the popularity of apps around the world. The number of smartphone users grew from 2.5 billion in 2016 to 3.5 billion in 2020 [13]. Keeping in line with this growth, the number of smartphone users accessing the internet with their phones has also increased. According to Statista [14], the penetration of internet users with mobile phones around the globe jumped from 52.7 percent in 2015 to 63.4 percent in 2019. Mobile apps have also advanced and grew over the years. Data suggests that the number of downloaded apps increased from 140 billion in 2016 to 204 billion in 2019 [15]. The most popular apps were games, ride, and taxis, video, and streaming, dating whereas health was one of the least popular along with music, banking, and news [16]. Privacy concerns are one of the main reasons for such a lower popularity level. Becker (2016) explained that during online treatment, sensitive data about the client is collected, which can lead to privacy and similar concerns about online applications. Thus, this might be a discouraging factor for clients comparing to using an online application or face-to-face treatment [17]. Although in small numbers people are accepting these apps, researchers have shown some significant benefits of using apps for mental health specifically.

Mobile apps were first launched in 2008 [18]. Around 325,000 health-related apps existed in 2017 [19]. This number is increasing continuously. According to Roth, these apps can be classified into mainly four categories: a) apps that provide health information; b) apps that help in diagnosis; c) apps that help in monitoring and controlling medicine; d) apps turn into a medical device by attaching extra equipment to it [20-21]. Health-related apps are covering many areas to provide services to both patients and doctors without visiting the doctors' offices. The same perspective applies to mental health apps as well. Mental health apps can provide information, build awareness, monitors mood and behavioral patterns. But the efficiency of the apps will depend on several factors as pointed by East and Havard [22] that well-designed mental health mobile apps that present content in interactive, engaging, and stimulating ways can promote cognitive learning, personal growth, and mental health enhancement. In the case of depression and other psychotic signs, mobile apps are seen as tools that help to collect data, to monitor, and also to conduct self-assessment $[18,23]$. Although there are some positive findings, challenges remain. The main concerns cited are the safety, standard, effectiveness of the apps as well as users' privacy [12]. Overall, mental health apps are generally accepted by users. Walsh and others found that, patients with eating disorders liked monitoring in theory but not in practice; people with depression and anxiety had lower rates of participation and believed technology-based symptom monitoring in its current forms had limited utility; and people with bipolar disorder viewed such monitoring more favorably [24].

The uses of Apps in health care are increasing phenomenally. The Apps and their uses in health care are a relatively new phenomenon, so are scholarships related to it. Scholars from various disciplines such as medicine, public health, psychology, engineering and communication are investigating these phenomena from multiple perspectives. Most of the scholarships focuses on the interventional aspects of mobile phone in mental health care. Little is known about different features of mental health related apps. Within such circumstances, this paper attempts to draw a map of existing mental health apps and its various dimensions.

\section{METHODOLOGY}

We collected data from Google play store between January 2016 and February 2020. We have chosen Google Play store purposively as its data are easily accessible and extractable. Moreover, the Apple store does not allow extracting data. We used a set of keywords to search apps available on the Google Play Store. The keywords include mental health, mental illness, mental disorders, and cure of mental disorder and healing of mental illness. Then we extracted data of the apps by using Beautiful Soup, a Python library for pulling data out of HTML and XML files. In this process, we amassed a huge stack of unstructured and messy data such as the name of the App, description of the app, price of the app, number of downloads, 
interactivity. We used a python library called Pandas to clean and structure the scraped data. By analyzing the keywords and descriptions of the apps, we categorized different types of apps for different mental illnesses and methods suggested for cure and healing. Then we created a data set by using some programming methods such as python programming language. We stored the data by using Sqlite3. Finally, we performed various descriptive statistical techniques to get the results. Our inclusion criteria were availability of data on the Apps, the Apps which used the English language in its description part, and dealt with common mental disorders. We excluded the Apps which did not deal with common mental disorders and in a non-English language.

\section{RESULTS}

Mobile phones are arguably one of the fastest spreading and adopted technologies across the globe. Various applications to perform specific tasks have made it a ubiquitous tool in human life. During the study period, we found a total of 837 Apps on mental illness available at Google Play Store. Based on their ease of use and functionality, any digital technology can be divided in two categories--static or not interactive and interactive. Interactive features allow a user to navigate platform tool easily. We found, as shown in the Table 1, that although 78.7\% Apps were not mentioned as interactive by the developers in the description of the Apps, they were actually embedded with various text, audio, video and other pictorials which made them interactive. The number of downloads is an important indicator of popularity and usefulness of the Apps. Meanwhile, 22.3\% Apps were mentioned as interactive.

The number of downloads is an important indicator of the usability and popularity of an App. As shown in Table 1, we found that the download ranged between 1-10000000 or more. We found that only two Apps were downloaded by users two times. In most cases, 33.2\% of Apps were downloaded for more than 1000 times by users. Meanwhile, 21.7\% Apps were downloaded for over 100 times and 10000 times respectively; $10.5 \%$ were downloaded for more than 100000 times, $4.5 \%$ were downloaded for more than 100000 times and $1.8 \%$ Apps were downloaded for more than 10000000 times. So, it is evident that most popular Apps are downloaded on average for a thousand times.

The financial cost works as a deciding factor that prevents an individual from seeking mental health care. They search for low cost or free tools as their pathway toward self-care of mental health. We found that 692 (82.7\%) Apps are freely available for users. Meanwhile, users are required to pay for only $146(17.4 \%)$ apps with additional features. We suspect that free availability is one of the main reasons behind the popularity of the Apps related to mental health issues.

Empirical evidence shows that a new user of an App looks at the rating and the number of people rated the App as a trust mechanism and deciding whether to download the App or not. In this study, we found, as shown in Table 1, that rating of the Apps ranged between 0 to 5. Only 2\% of Apps were rated 0-1. Meanwhile, 3.0\% Apps were rated 4-5, 75.1\% were 3-4 and 19.6\% were rated 2-3. So, it can be argued that the average rating of the most popular Apps ranged between 3 to 5. On the other hand, the number of people who rated the Apps reflects the users' satisfaction level and opinion about a certain App. The data in Table 1 show that the number of people who rated the Apps ranged between 0 to 1000000 . In most cases $(39.2 \%)$, Apps were rated by only 0-10 users and $31.1 \%$ Apps were rated by only 10-100 users. It clearly indicates that most mental health Apps users do not leave their opinion or share their experience with the App. So, it would be difficult to predict the trustworthiness or popularity of mental health-related Apps by looking at their rating and the number of people who rated.

As mentioned earlier that different common mental disorders affect the lives of people of different age groups. Keeping this in mind, App developers target people of a minimum three years of age and a maximum of over 18 years. We found that the developers of the Apps built those tools in such a way that it is child friendly. We found that $95.5 \%$ of Apps were targeted for the individual of at least three years of age.

We categorized the Apps available on Google Play Store based on their applicability to various mental health issues. As shown in the Table 2, we found that $26.4 \%$ deal with general mental health, $22.7 \%$ with depression, $17.8 \%$ anxiety, $26.4 \%$ general mental health, $9.0 \%$ stress, $3.6 \%$ post-traumatic disorder, $2.3 \%$ 
bipolar disorder, $1.6 \%$ panic disorder, $7.5 \%$ sleep disorder, $2.4 \%$ schizophrenia, $3.5 \%$ obsessive-compulsive disorder, $1.4 \%$ substance abuse, $1.2 \%$ addiction.

Table 1: Apps' interactivity, number of download, prices, rating, number of people rated Apps and target age group

\begin{tabular}{|c|c|c|}
\hline \multicolumn{3}{|c|}{ Interactivity } \\
\hline Not Stated as Interactive & 650.0 & $78.7 \%$ \\
\hline Stated as Interactive & 188.0 & $22.3 \%$ \\
\hline \multicolumn{3}{|c|}{ Number of downloads } \\
\hline $1+$ & 2.0 & $0.2 \%$ \\
\hline $10+$ & 52.0 & $6.2 \%$ \\
\hline $100+$ & 182.0 & $21.7 \%$ \\
\hline $1000+$ & 278.0 & $33.2 \%$ \\
\hline $10000+$ & 182.0 & $21.7 \%$ \\
\hline $100000+$ & 88.0 & $10.5 \%$ \\
\hline $100000+$ & 38.0 & $4.5 \%$ \\
\hline $10000000+$ & 15.0 & $1.8 \%$ \\
\hline \multicolumn{3}{|c|}{ Prices of App } \\
\hline Paid & 146.0 & $17.4 \%$ \\
\hline Free & 692.0 & $82.7 \%$ \\
\hline \multicolumn{3}{|c|}{ User Rating } \\
\hline $0-1$ & 10.0 & $1.2 \%$ \\
\hline $1-2$ & 10.0 & $1.2 \%$ \\
\hline $2-3$ & 164.0 & $19.6 \%$ \\
\hline $3-4$ & 629.0 & $75.1 \%$ \\
\hline $4-5$ & 25.0 & $3.0 \%$ \\
\hline \multicolumn{3}{|c|}{ Number of users gave ratings } \\
\hline $0-10$ & 328.0 & $39.2 \%$ \\
\hline $10-100$ & 260.0 & $31.1 \%$ \\
\hline $100-1000$ & 126.0 & $15.1 \%$ \\
\hline $1000-10000$ & 68.0 & $8.1 \%$ \\
\hline $10000-100000$ & 35.0 & $4.2 \%$ \\
\hline $100000-1000000$ & 21.0 & $2.5 \%$ \\
\hline \multicolumn{3}{|c|}{ Age group } \\
\hline $3+$ & 799.0 & $95.5 \%$ \\
\hline $12+$ & 30.0 & $3.6 \%$ \\
\hline $16+$ & 7.0 & $0.8 \%$ \\
\hline $18+$ & 2.0 & $0.2 \%$ \\
\hline
\end{tabular}

The Apps offered various approaches to improve the mental health conditions seen in Table 3. The approaches include relaxation (31.4\%), stress management (13.5\%), symptom tracking (22.6\%), calming audio $(11.7 \%)$, keeping diary $(4.9 \%)$, connect with mental health resources $(1.1 \%)$, interpersonal support (2.7\%), meditation (2.6\%) and mood tracking (9.4\%). According to data shown in Table 3, most of the Apps offered various relaxation methods for improving mental health, followed by symptom tracking, calming audio, and mood tracking.

Average rating and number of downloads are important indicators of the popularity of an App. As shown in Table 4, we found that the average rating of the Apps meant for depression was 3.71 and total downloads were 119172625 during the study period. Meanwhile, the average rating for the Apps related anxiety was 4.07 and the total download was 128681115 . The data in the table shows that Apps related to Addiction got a higher level of ratings (4.39). The data in the table show that Apps related to general mental health, bipolar 
disorder, Sleep disorder, obsessive-compulsive disorder, substance abuse, and addiction received rating over 4.

Table 2: Mental health diagnosis or symptoms mentioned

\begin{tabular}{|c|c|c|}
\hline Symptoms & $\mathbf{N}$ & $\mathbf{\%}$ \\
\hline Depression & 190 & 22.7 \\
\hline Anxiety & 149 & 17.8 \\
\hline General Mental Health & 221 & 26.4 \\
\hline Stress & 75 & 9.0 \\
\hline Post traumatic stress disorder & 30 & 3.6 \\
\hline Bipolar disorder & 19 & 2.3 \\
\hline Panic disorder & 13 & 1.6 \\
\hline Sleep disorder & 63 & 7.5 \\
\hline Schizophrenia & 20 & 2.4 \\
\hline Obsessive compulsive disorder & 29 & 3.5 \\
\hline Addiction (Non drug and alcohol related addiction) & 12 & 1.4 \\
\hline No specific symptom or diagnosis mentioned & 10 & 1.2 \\
\hline Total & 6 & 0.7 \\
\hline
\end{tabular}

Table 3: Approaches to improving mental health used by the app

\begin{tabular}{|c|c|c|}
\hline Approaches to improving mental health & $\mathbf{N}$ & $\mathbf{\%}$ \\
\hline Relaxation & 263 & 31.4 \\
\hline Stress management & 113 & 13.5 \\
\hline Symptom tracking & 189 & 22.6 \\
\hline Calming audio & 98 & 11.7 \\
\hline Keeping diary & 41 & 4.9 \\
\hline Connect with mental health resources & 9 & 1.1 \\
\hline Interpersonal support & 23 & 2.7 \\
\hline Meditation & 22 & 2.6 \\
\hline Mood tracking & 79 & 9.4 \\
\hline Total & 837 & 100.0 \\
\hline
\end{tabular}

Table 4: Rating and number of downloads of mental illness Apps

\begin{tabular}{|c|c|c|c|}
\hline Symptom mentioned & Average Rating & No. of people that rated & No. of downloads \\
\hline Depression & 3.7 & 2823087 & 119172625 \\
\hline Anxiety & 4.0 & 4791981 & 128681115 \\
\hline General Mental Health & 4.2 & 933154 & 509345250 \\
\hline Stress & 3.9 & 115726 & 6237220 \\
\hline Post traumatic stress disorder & 3.9 & 4525421 & 138855800 \\
\hline Bipolar disorder & 4.0 & 4542456 & 122233715 \\
\hline Panic disorder & 3.5 & 1902845 & 566562125 \\
\hline Sleep disorder & 4.0 & 1981421 & 564272180 \\
\hline Schizophrenia & 3.6 & 123580 & 4157810 \\
\hline Obsessive compulsive disorder & 4.0 & 708895 & 29081195 \\
\hline Substance abuse (Drug and Alcohol) & 4.0 & 86551 & 3808520 \\
\hline Addiction (Technology etc.) & 4.3 & 62389 & 2294900 \\
\hline
\end{tabular}


Data in Table 4 show that keeping diary related Apps got higher ratings. The rating of Apps related to relaxation, stress management, symptom tracking, and calming audio was higher than other apps. But interestingly the Apps got relatively less rating by the users as shown in Table 5 .

Table 5: Average rating and number of downloads of the Apps in relations to approaches to improving mental health

\begin{tabular}{|c|c|c|c|}
\hline Approaches & Average Rating & No. people who rated & No. of downloads \\
\hline Relaxation & 3.9 & 2243400 & 576704190 \\
\hline Stress management & 3.9 & 2236746 & 544899540 \\
\hline Symptom tracking & 3.8 & 429950 & 17048120 \\
\hline Calming audio & 3.9 & 4183295 & 111331280 \\
\hline Keeping diary & 4.0 & 4811544 & 630345215 \\
\hline $\begin{array}{c}\text { Connect with mental health } \\
\text { resources }\end{array}$ & 3.4 & 371209 & 30248490 \\
\hline Interpersonal support & 3.2 & 6638452 & 669650610 \\
\hline Meditation & 3.9 & 17431 & 1249960 \\
\hline Mood tracking & 3.9 & 1064131 & 62793525 \\
\hline
\end{tabular}

\section{DISCUSSION}

This study was a content analysis of mental health-related Apps available on Google Play Store. Our results show that most of the Apps deal with some common mental disorders such as depression, anxiety, and general mental health. Meanwhile, the Apps offer some behavioral interventions such as symptom tracking, mood tracking, stress management calming audio, meditation, and interpersonal support. Although these apps are not directly or indirectly endorsed by the medical practitioners, they are working to provide support to ease the mental disorder and bring mental wellness. For example, Vázquez et al. (2018) argued that mobile apps have a high potential to deliver mental health interventions, especially for depression [25]. But clinicians should cautiously incorporate the apps as an auxiliary to treatment or recommending apps to clients [26]. Because, some App may not bring desired results, rather may prove to be iatrogenic for some patients.

Features and functionalities of mental health apps are many which create interest among the app users to install and use an app. We found that most of the Apps have interactive features. But the effects of the Apps depend on how users interact with mental health apps in clinically meaningful ways. Zhang et al., (2019) argued that clinically meaningful activities-learning, goal setting, and self-tracking-may reduce depression symptoms of a user [27].

Our results show that most popular Apps are downloaded on average for a thousand times. Multiple factors that boost application download include high ratings, frequent updating, long-standing market presence [28]. But a high rate of download of mental health apps does not automatically translate into high use [29]. Our findings indicate that most mental health Apps users do not leave their opinion or share their experience with the App. So, it would be difficult to predict the trustworthiness or popularity of mental-health related Apps by looking at their rating and the number of people who rated. Mahmood (2019) examined the influence of various factors of apps on google play apps ratings [30]. The researcher found that there are some words promise higher ratings while there are some keywords that usually mean lower ratings.

There are often questions raised about the acceptance and effectiveness of mental health-related apps. This concern was visible in the number of downloads as a majority of the apps were downloaded for only a thousand times. Such a small number of downloads indicate that people are accepting these apps in modesty. Some of the popular apps are being downloaded more than a million at present. For instance, gaming apps such as Call of Duty was installed 170 million times [31]. But the developers of mental health apps are trying to encourage wider use. They made these apps for all age groups and free of cost. This suggests that the 
majority of the mental health apps are made simple, easy to use, and involves less complex interactive healing method which is suitable for all. Hence these apps are easily accessible, portable, and cost-effective. Our results show that $82.7 \%$ of Apps are freely available for users. Meanwhile, users are required to for only $17.4 \%$ Apps which have a bit more functionality than the freely available Apps. Free availability might be a reason behind the popularity of the Apps.

\section{CONCLUSIONS}

Mental health Apps mainly deal with following symptoms: depression, anxiety, general mental health, stress, post-traumatic stress disorder, bipolar disorder, panic disorder, sleep disorder, schizophrenia, obsessive-compulsive disorder, substance abuse (drug and alcohol), addiction (non-drug and alcohol-related addiction). On the other hand, Apps offer various approach to improve mental health. The approaches include relaxation, stress management, symptom tracking, calming audio, keeping a diary, connect with mental health resources, interpersonal support, meditation, and mood tracking.

Most Apps related to mental health are easy to use and interactive. Most popular Apps are downloaded on average for a thousand times. Moreover, our findings indicate the reasons behind the popularity of Apps related to mental health issues as those are freely available. It would be difficult to predict the trustworthiness or popularity of mental health-related Apps by looking at their rating and the number of people who rated. We found that most of the Apps were targeted for an individual of at least three years of age.

The main limitation of the study is that it mainly dealt with the Apps available at Google Play Store. No other App markets were investigated. It also did not investigate the impact and effectiveness of the Apps as a tool for psychological and behavioral intervention. Future research should focus on the effectiveness of the Apps to improve mental health.

Mental health Apps is a relatively new phenomenon, in dealing with mental health and mental disorder. The results found in this research would be of interest to App developers to consider when creating a health behavior app. It would also help to physicians and general users. Future research should focus on the effectiveness of the Apps to improve mental health.

\section{REFERENCES}

1. WHO. Depression and Other Common Mental Disorders: Global Health Estimates. Geneva, Switzerland: WHO Document Production Services; 2017.

2. Vigo D, Thornicroft G, Atun R. Estimating the true global burden of mental illness. Lancet Psychiatry 2016;3(2):171-8.

3. Gustavson K, Knudsen AK, Nesvåg R, Knudsen GP, Vollset SE, Reichborn-Kjennerud T. Prevalence and stability of mental disorders among young adults: findings from a longitudinal study. BMC Psychiatry 2018;18(1).

4. Kendrick T, Pilling S. Common mental health disorders - identification and pathways to care: NICE clinical guideline. Br J Gen Pract 2012;62(594):47-9.

5. Steel Z, Marnane C, Iranpour C, Chey T, Jackson JW, Patel V, et al. The global prevalence of common mental disorders: a systematic review and meta-analysis 1980-2013. Int J Epidemiol 2014;43(2):476-93.

6. Knaak S, Mantler E, Szeto A. Mental illness-related stigma in healthcare. Health Manage Forum 2017;30(2):111-6.

7. Anderson K, Burford O, Emmerton L. Mobile Health Apps to Facilitate Self-Care: A Qualitative Study of User Experiences. Plos One 2016;11(5).

8. Corrigan P. How stigma interferes with mental health care. Amer Psychol 2004;59(7):614-25.

9. Kenny R, Dooley B, Fitzgerald A. Developing mental health mobile apps: Exploring adolescents' perspectives. Health Inform J 2016;22(2):265-75.

10. Bakker D, Kazantzis N, Rickwood D, Rickard N. A randomized controlled trial of three smartphone apps for enhancing public mental health. Behav Res Ther 2018;109:75-83.

11. Bakker D, Rickard N. Engagement in mobile phone app for self-monitoring of emotional wellbeing predicts changes in mental health: MoodPrism. J Affect Disord 2018;227:432-42.

12. Marley J, Farooq S. Mobile telephone apps in mental health practice: uses, opportunities and challenges. BJ Psych Bull 2015;39(6):288-90.

13. Statista. Number of smartphone users worldwide from 2016 to 2021 (in billions). [Internet]. Statista. 2020 [cited 2020Mar2]. Available from: https://www.statista.com/statistics/330695/number-of-smartphone-usersworldwide/ 
14. Statista. Mobile phone internet user penetration worldwide from 2014 to 2019 [Internet]. Statista. 2020 [cited 2020Feb17]. Available from: https://www.statista.com/statistics/284202/mobile-phone-internet-userpenetration-worldwide/

15. Statista. Number of mobile app downloads worldwide from 2016 to 2019(in billions) [Internet]. Statista. 2020 [cited 2020Mar17]. Available from: https://www.statista.com/statistics/271644/worldwide-free-and-paidmobile-app-store-downloads/

16. Statista. Mobile app verticals with the highest install and user base growth in 2018 [Internet]. Statista. 2019 [cited 2020Mar17]. Available from: https://www.statista.com/statistics/251096/fastest-growing-shoppingapp-categories/

17. Becker D. Acceptance of Mobile Mental Health Treatment Applications. Proc Comp Sci 2016;98:220-7.

18. Donker T, Petrie K, Proudfoot J, Clarke J, Birch M-R, Christensen H. Smartphones for Smarter Delivery of Mental Health Programs: A Systematic Review. J Med Internet Res 2013;15(11):1-13.

19. Research2Guidance. 325000 mobile health apps available in 2017- Android now the leading mHealth platform. [Internet]. research2guidance. 2017 [cited 2020Mar17]. Available from: https://research2guidance.com/325000-mobile-health-apps-available-in-2017/

20. Roth VJ. The mHealth Conundrum: Smartphones \& Mobile Medical Apps - How Much FDA Medical Device Regulation Is Required. North Carol J Law Technol 2014;15(3):359-424.

21. Larson RS. A Path to Better-Quality mHealth Apps. JMIR mHealth and uHealth 2018;6(7):1-5.

22. East ML, Havard BC. Mental Health Mobile Apps: From Infusion to Diffusion in the Mental Health Social System. JMIR Mental Health 2015;2(1):1-14.

23. Palmier-Claus JE, Ainsworth J, Machin M, Barrowclough C, Dunn G, Barkus E, et al. The feasibility and validity of ambulatory self-report of psychotic symptoms using a smartphone software application. BMC Psychiatry 2012;12(1):1-10.

24. Walsh S, Golden E, Priebe S. Systematic review of patients' participation in and experiences of technologybased monitoring of mental health symptoms in the community. BMJ Open 2016;6(6):1-9.

25. Vázquez FL, Torres Á, Díaz O, Páramo M, Otero P, Blanco V. Cognitive behavioral intervention via a smartphone app for non-professional caregivers with depressive symptoms: study protocol for a randomized controlled trial. Trials 2018;19(1).

26. Lui JHL, Marcus DK, Barry CT. Evidence-based apps? A review of mental health mobile applications in a psychotherapy context. Profess Psychol Res Pract 2017;48(3):199-210.

27. Zhang R, Nicholas J, Knapp AA, Graham AK, Gray E, Kwasny MJ, et al. Clinically Meaningful Use of Mental Health Apps and its Effects on Depression: Mixed Methods Study. J Med Internet Res 2019;21(12).

28. Krishnan G, Selvam G. Factors influencing the download of mobile health apps: Content review-led regression analysis. Health Pol Technol 2019;8(4):356-64.

29. Torous J, Wisniewski H, Liu G, Keshavan M. Mental Health Mobile Phone App Usage, Concerns, and Benefits Among Psychiatric Outpatients: Comparative Survey Study. JMIR Mental Health. 2018;5(4).

30. Mahmood A. Identifying the influence of various factor of apps on google play apps ratings. J Data Inform Manage 2019;2(1):15-23

31. Jones K. Ranked: The World's Most Downloaded Apps [Internet]. Visual Capitalist. 2020 [cited 2020Mar17]. Available from: https://www.visualcapitalist.com/ranked-most-downloaded-apps/.

$* * * * * * * * * * * * * * * * * * * * * * * * * * * * * * * * * * * *$

Acknowledgements - Nil.

Conflict of Interest - Nil

Funding - Nil 